\title{
The Effect of Reversed Loading Conditions on the Mechanical Behaviour of Extruded Magnesium Alloy AZ31
}

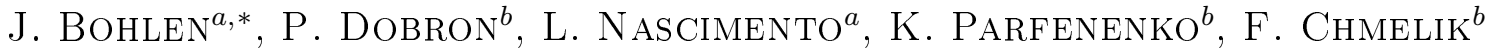 \\ AND D. LETZIG ${ }^{a}$ \\ ${ }^{a}$ Magnesium Innovation Centre, Helmholtz-Zentrum Geesthacht, D21502 Geesthacht, Germany \\ ${ }^{b}$ Department of Physics of Materials, Charles University, Ke Karlovu 5, CZ 12116 Prague 2, Czech Republic

\begin{abstract}
An indirectly extruded round bar of magnesium alloy AZ31 has been subjected to a cyclic test consisting
\end{abstract} \\ of preloading in compression to different values of maximum stress followed by a single tensile test segment. \\ Concurrent acoustic emission measurements were used to determine the active deformation mechanisms during \\ plastic flow and work hardening. Electron backscattering diffraction was applied to obtain local orientation \\ images in order to reveal twins and twinned fractions of the microstructure. Twins form preferentially in \\ larger grains during the compression test segment and only with increasing stress do smaller grains show \\ twinning. Some grains are completely re-oriented as a result of twinning. During the tensile test segment, \\ untwinning is the most significant deformation mechanism although in some re-oriented grains new twins also nu- \\ cleate. The acoustic emission count rates confirm that this is only the case after compression to higher stress levels.
}

PACS: 43.40.Le, 61.72.Mm, 81.05.Bx, 81.70.Bt

\section{Introduction}

The mechanical properties of extruded magnesium alloys typically exhibit a distinct tension-compression asymmetry in the yielding and work hardening behaviour [1-3]. The yield stress is significantly lower in compression than in tension. Furthermore, a very low work hardening rate is found at the beginning of the compression test, which increases to a maximum with increasing strain before decreasing again at higher strains. During tensile loading, on the other hand, the initial work hardening rate is high and typically decreases with increasing strain $[1,4,5]$. This behaviour is associated with the asymmetric activation of twinning in textured magnesium alloys. Twins of the $\{10-12\}\langle 10-1-1\rangle$ type are favoured if the stress is applied in tension parallel to the $c$-axis of the hcp lattice [6]. However, the texture of extruded bars is characterised by the preferential orientation of the basal planes parallel to the extrusion direction so that this type of twinning is preferentially activated in compression along the extrusion axis rather than in tension [3].

It has been shown in a number of studies that the activation of twinning depends on a number of microstructural features besides the texture. Firstly, the yield asymmetry decreases with decreasing average grain size [7]. This is understood to result from an increase of the twinning stress with decreasing grain size, which leads to a more distinct increase of the yield stress if the plastic flow is twin dominated $[1,8]$. Secondly, the grain size

\footnotetext{
* corresponding author; e-mail: jan.bohlen@hzg.de
}

distribution plays an important role for the activation of twinning. Dobron et al. [9] have shown that larger grains tend to twin preferentially compared to smaller grains. New twins form in the strain regime where an increasing work hardening rate is found, i.e. as the slope of the stress-strain curve increases. After this point, a decrease in the slope correlates with twin growth but no further formation of new twins.

A considerable number of applications require knowledge of the behaviour of materials under cyclic loading. The asymmetric activation of twinning in different grain size fractions therefore requires a more detailed analysis. If a compressive cycle segment is followed by a tensile cycle segment, a distinct change in the material behaviour is observed showing a flow behaviour which is typical for twin dominated deformation [4, 5, 10]. This behaviour has been basically correlated with untwinning during this tensile deformation segment.

The acoustic emission (AE) technique can be used as an in situ method for the detection of transient elastic waves generated within the material during deformation due to sudden localised structural changes. Such an analysis is carried out using differences in the amplitudes of the respective signals, i.e. different amplitude levels for counting are applied [11]. A continuous AE with lower amplitude is typically the result of dislocation glide whereas burst type emission with higher amplitudes is the result of massive dislocation glide, twinning or local cracking [7, 12].

In this paper, the influence of compressive pre-straining on a subsequent tensile test segment is examined, in order to reveal the grain size dependences and 
twinning activities during testing. The concurrent measurement of the $\mathrm{AE}$ is used to distinguish between twin nucleation, twin growth and untwinning.

\section{Experimental}

In this study, a round bar of the magnesium alloy AZ31 $(\mathrm{Mg}+3 \mathrm{wt} \% \mathrm{Al}+1 \mathrm{wt} \% \mathrm{Zn}+0.3 \mathrm{wt} \% \mathrm{Mn})$ was used, which had been indirectly extruded at $300^{\circ} \mathrm{C}$ with an extrusion rate (profile exit speed) of $5 \mathrm{~m} / \mathrm{min}$. The extrusion ratio was 1:30. Specimens with a cylindrical geometry with screws on both ends having their axes parallel to the extrusion direction were prepared by machining. The gauge length was $15 \mathrm{~mm}$ and the diameter $8 \mathrm{~mm}$. Mechanical tests were carried out at ambient temperature using a universal testing machine (Zwick Z050). The strain rate was constant at $0.02 \mathrm{~mm} / \mathrm{s}$. The maximum stress used for the compression test segment was varied between 125 and $200 \mathrm{MPa}$; maximum stresses used in the subsequent tensile test segment were up to at least $250 \mathrm{MPa}$. The tests were stopped prior to sample fracture. Additionally, mechanical tests were repeated and stopped at distinct points in order to carry out microstructure analysis.

A computer-controlled DAKEL-CONTI-4 acoustic emission system was used to monitor AE during the mechanical tests based on a continuous sampling of the AE signals. Four channels with varying amplification and $2 \mathrm{MHz}$ sampling frequency are available to detect and store data on a hard disc. For the data presented in this paper an amplification of $35 \mathrm{~dB}$ was used. The results presented in this work are extracted on the basis of a standard two threshold-level detection procedure [13]. More details on the data analysis are reported in earlier work [14].

Electron backscattering diffraction (EBSD) was used to analyse the microstructure and texture of the samples in a field emission gun scanning electron microscope (Zeiss Ultra 55, EDAX/TSL EBSD system and Hikari detector) on electropolished longitudinal sections of the above mentioned samples.

\section{Results}

Figure 1 shows the results of three cyclic loading tests with concurrent $\mathrm{AE}$ count rates as a function of the measuring time. The first segment of the measurements represents the compression test segment (shown as positive stress) up to a maximum stress which is $125 \mathrm{MPa}$ in Fig. 1a, $150 \mathrm{MPa}$ in Fig. 1b and $200 \mathrm{MPa}$ in Fig. 1c. During this test segment, yielding occurs at a compressive yield stress of $120 \mathrm{MPa}$ and plastic flow continues with an increasing slope of the flow curve. Following the compression test segment, there is a continuous decrease of compressive stress to zero before the tensile test segment starts (shown as negative stress). The flow behaviour of the material during tensile loading varies as a function of the maximum stress of the prior compression test segment. After a low compressive stress and corresponding low strain in compression (Fig. 1a), a continuous decrease of the slope is found. In Fig. 1b and c, however, the flow curve shows more similarity to that of the compression test segment with an increase of the slope which is then followed by a decrease again. The higher the stress the sample received in compression, the more significant is this behaviour.

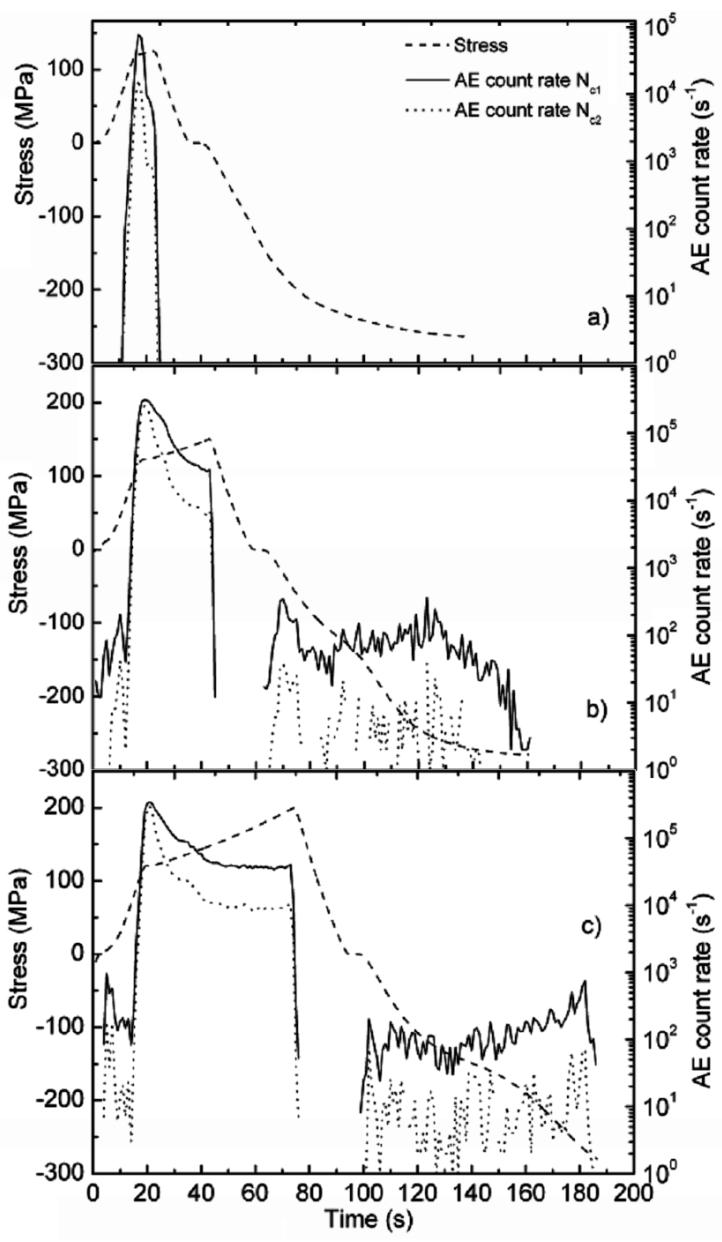

Fig. 1. Stress diagram and AE count rates for cyclic tests with maximum compressive stresses of (a) $125 \mathrm{MPa}$, (b) $150 \mathrm{MPa}$ and (c) $200 \mathrm{MPa}$; compressive stress is plotted positive and tensile stress is plotted negative; $N_{\mathrm{c} 1}$ is the $\mathrm{AE}$ count rate of continuous $\mathrm{AE}$ at lower threshold level and $N_{\mathrm{c} 2}$ is the AE count rate of burst type signals.

Figure 2 shows this in more detail, where all three tensile test segments are shown as stress-strain diagrams. The yield point is significantly reduced if the prior compressive pre-straining was higher. However, the samples subjected to compressive stresses of $150 \mathrm{MPa}$ and $200 \mathrm{MPa}$ show comparable behaviour up to the onset of plastic flow in tension, whereas the work hardening behaviour is distinctly different. The higher the prior compressive stress, the higher is the strain in tension at which the work hardening rate starts to increase. All three curves then develop up to a point where the slope de- 


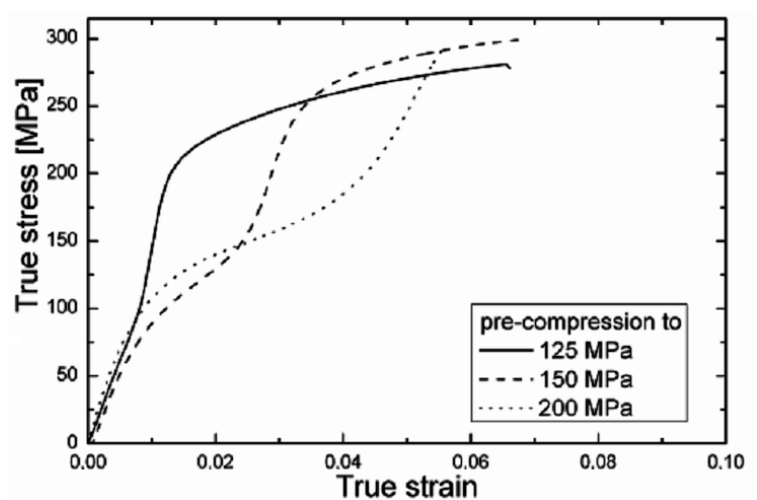

Fig. 2. Stress-strain curves of the tensile test segment after compression to different values of stress.

creases again and the subsequent flow behaviour is quite similar in all three experiments.

Figure 1 also shows the AE count rates at two threshold levels $N_{\mathrm{c} 1}$ and $N_{\mathrm{c} 2}$. During the compression segment of the test the AE count rates increase to a maximum where almost all signals are of the burst type. Only in Fig. 1a where the test segment is stopped at $125 \mathrm{MPa}$ close to the yield stress, this overall maximum could not be reached. Still, in Fig. $1 \mathrm{~b}$ and $\mathrm{c}$ the peak intensity is followed by a decrease in count rates at both threshold values up to the end of the respective test segment. During the subsequent tensile test segment, no AE was detected in Fig. 1a after the lowest pre-straining in compression. With higher levels of prior strain, as in Fig. 1b and $c$, low and continuous AE count rates are found at both threshold levels. However, these count rates are significantly lower than during the initial compression test segment.

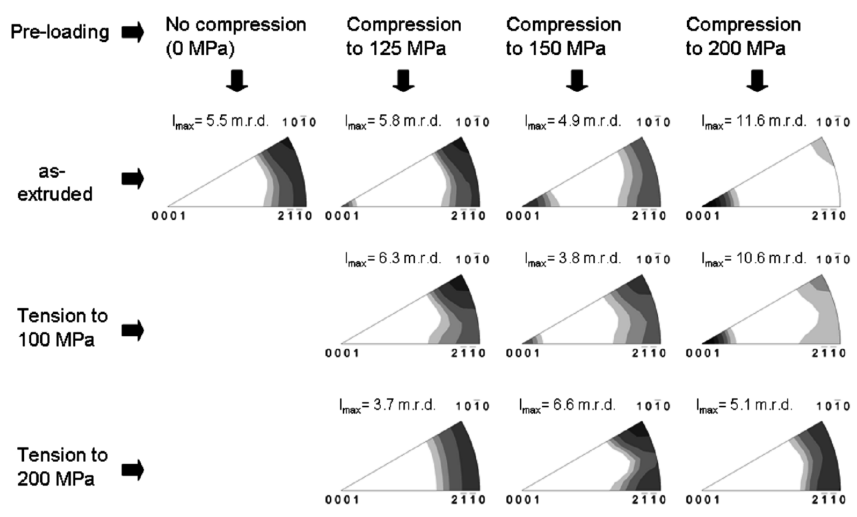

Fig. 3. Inverse pole figures in the extrusion direction of samples after compression to different values of stress and subsequent tensile testing.

Figure 3 shows inverse pole figures in the extrusion direction which is also the testing direction. The initial texture of the extruded material exhibits a typical $\langle 1010\rangle-\langle 11-20\rangle$ texture with a higher intensity near the $\langle 10-10\rangle$-pole. Such a texture is often found in magnesium extrusions $[3,15]$ and basically reveals the preferential orientation of basal planes parallel to the extrusion direction. The inverse pole figures measured after the compression test segment (first row in Fig. 3) show an increasing intensity of the component at the $\langle 0001\rangle$ pole at the expense of the original $\langle 10-10\rangle-\langle 11-20\rangle$ orientations. Thus, a continuous re-orientation of the microstructure is found which is more significant with increasing compressive stress. In the second row in Fig. 3, the pole figures after the subsequent tensile test segment up to $100 \mathrm{MPa}$ are shown. Generally, a decrease in the $\langle 0001\rangle$ pole intensity is found, which leads to re-orientation to the $\langle 10-10\rangle$ - and $\langle 11-20\rangle$-poles again. The higher the initial compressive stress, the higher is the tensile stress required for complete disappearance of the $\langle 0001\rangle$ component, as seen in the pole figures in row 3 in Fig. 3 after tensile testing to $200 \mathrm{MPa}$. There is no distinct difference between the original texture and the texture that develops as a result of the cyclic test segments.

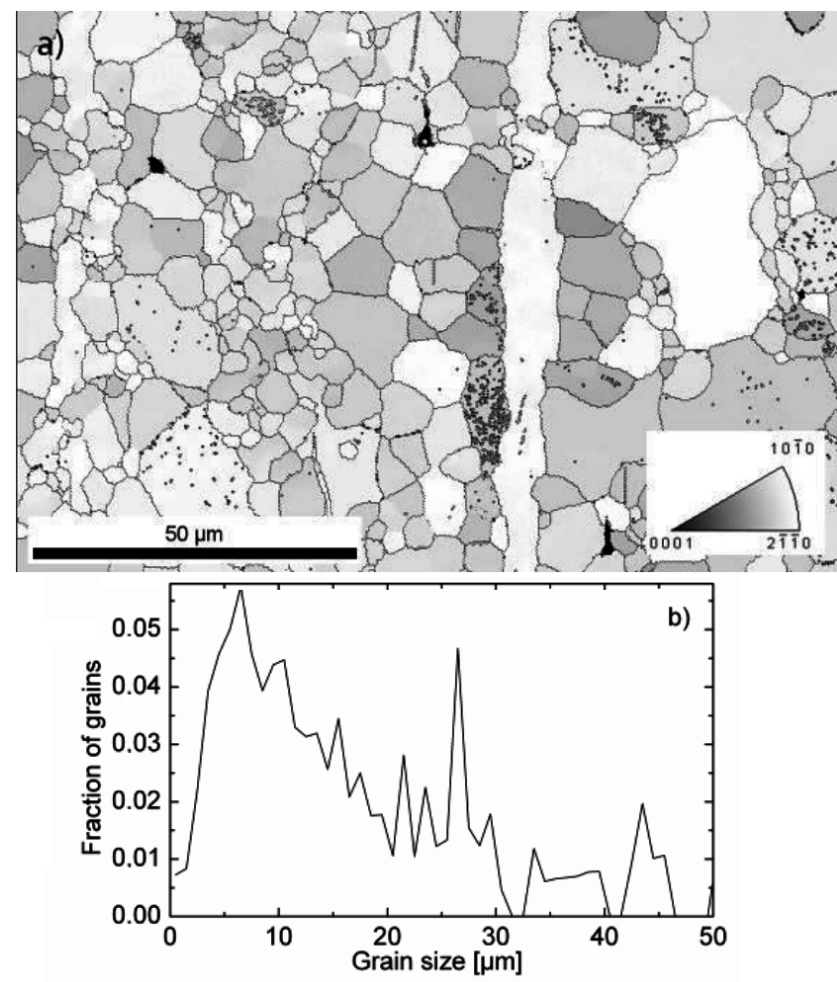

Fig. 4. Microstructure of the extruded round bar used for the test; (a) orientation map (colour indicates angle to $\langle 0001\rangle$-direction) and (b) grain size distribution.

Figure $4 \mathrm{a}$ shows an orientation map of the extruded bar before testing. The microstructure is characterised by a broad grain size distribution, as documented in Fig. 4b, with large elongated grains and a broader variation of equiaxed grains. The larger elongated grains are known to be unrecrystallised grains and typically ex- 


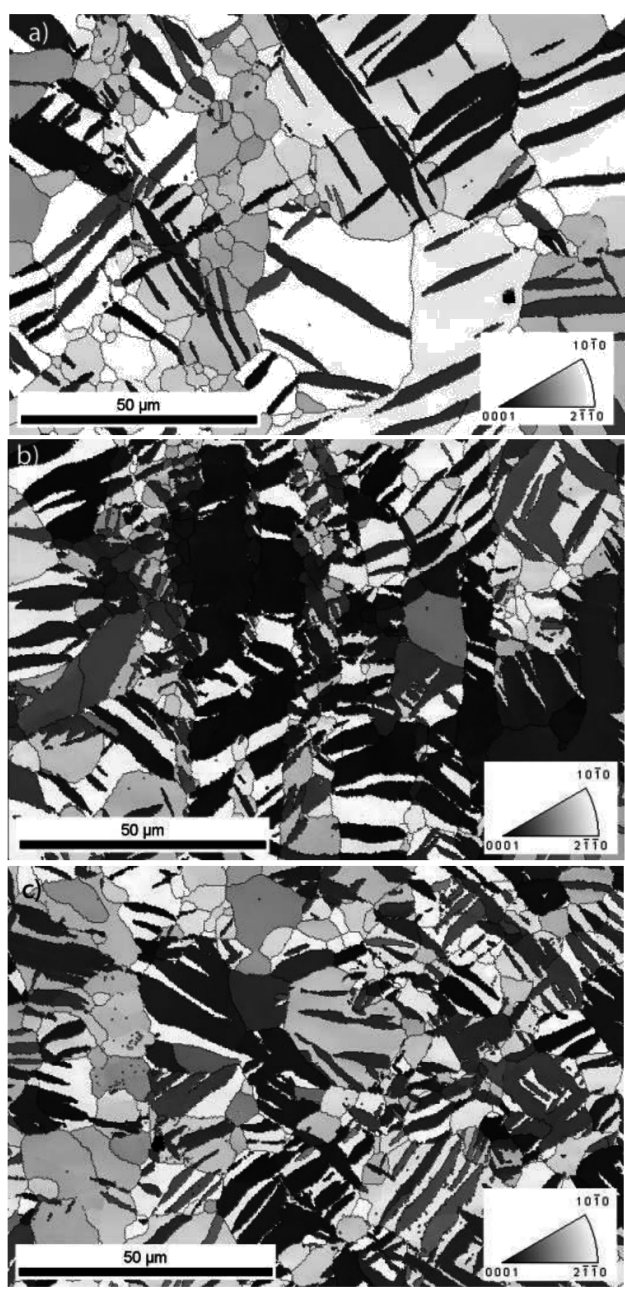

Fig. 5. Orientation maps of the extruded round bar after different test segments: (a) compression to $150 \mathrm{MPa}$, (b) compression to $200 \mathrm{MPa}$ and (c) compression to $200 \mathrm{MPa}$ and tension to $100 \mathrm{MPa}$, colour scale is the same as in Fig. 4.

hibit a strong $\langle 10-10\rangle$ orientation. The recrystallised, equiaxed grains typically show orientations along the arc between the $\langle 10-10\rangle$ - and the $\langle 11-20\rangle$-poles. The average grain size is $18 \mu \mathrm{m}$ with the highest fraction of grains being below this average size and large grains that can reach up to $50 \mu \mathrm{m}$.

Figure 5a shows the orientation map of a sample tested to $150 \mathrm{MPa}$ in compression. The re-oriented fractions of the microstructure can be identified as twins that are spread all over the microstructure. It is noted that larger grains, in particular, can contain several twins, obviously also with different directions of nucleation. Figure $5 \mathrm{~b}$ shows the same after applying a compressive stress of $200 \mathrm{MPa}$. In this condition, the twins became larger and in some cases lead to complete re-orientation of whole grains. Interestingly, these grains are of intermediate size. Twin boundaries are still identified in larger grains and smaller grains do not show any twins. Figure $5 \mathrm{c}$

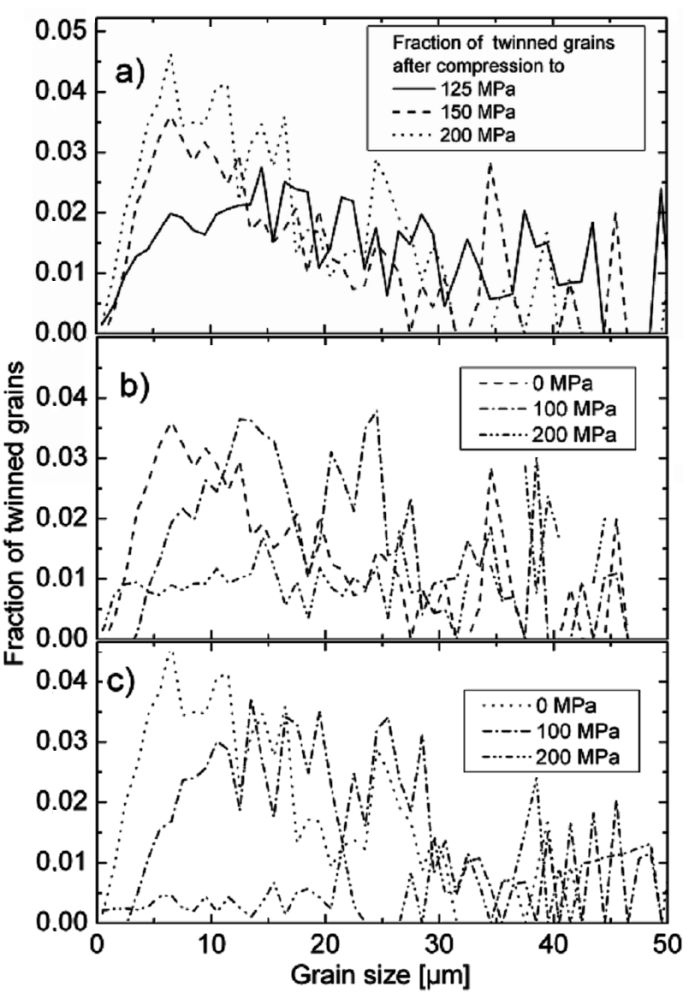

Fig. 6. Fraction of grains that contain twins or are completely twinned (a) after different compressive stresses, (b) after compression to $150 \mathrm{MPa}$ and different tensile stresses, (c) after compression to $200 \mathrm{MPa}$ and different tensile stresses.

shows the orientation map of a sample which received an additional $100 \mathrm{MPa}$ tensile stress after compression to $200 \mathrm{MPa}$. On the one hand, the twins in larger grains appear smaller again compared to the sample shown in Fig. 5b. On the other hand, in some grains twins appear with a $\langle 10-10\rangle$ - or $\langle 11-20\rangle$-orientation in a grain with an orientation close to the $\langle 0001\rangle$-pole. This type of twin is not found very frequently. However, this shows the possibility of such a mechanism.

Figure 6 a shows the fraction of grains that contain one or more twins as a function of the grain size. The analysis software used allows the twin boundaries to be ignored and those grains that contain twins, or are completely re-oriented as a result of twinning, to be marked. This distribution is shown for samples after different compressive stresses, revealing that in the beginning of the compression test larger grains tend to twin preferentially over smaller grains. Only with increasing stress does a larger fraction of smaller grains, which are generally found more frequently in the microstructure (see Fig. 4b), tend to twin as well, revealing a grain size dependence of twin formation. Figure $6 \mathrm{~b}$ and $\mathrm{c}$ show the development of the fraction of twinned grains with increasing tensile stress after compression testing to $150 \mathrm{MPa}$ and $200 \mathrm{MPa}$, respectively. Interestingly, the fraction of smaller grains that contain twins decreases significantly, whereas in the 
larger grains twins remain visible. At the lowest compressive stress of $125 \mathrm{MPa}$ (not shown), twins disappear almost completely if a tensile stress of $100 \mathrm{MPa}$ is applied, which can be understood with the initially small fraction of twins in this condition.

\section{Discussion}

Activation of the most common twinning mode $\{10-12\}\langle 10-1-1\rangle$ in magnesium $[6,16]$ is observed during compression testing of the extruded round bars. This is well illustrated in Fig. 5a and $\mathrm{b}$ and confirmed by the yielding and work hardening behaviour during the compressive test segment shown in Fig. 1a-c. On the other hand, twinning is not preferentially activated if the stress is initially applied in tensile testing as e.g. shown by $[1,4,7]$. However, during the tensile test segment of samples which were subjected to compressive pre-straining, the stress-strain curves show the typical compression test form. The higher the initial compressive stress, i.e. the higher the fraction of twinned microstructure, the more significant is this behaviour, see Fig. 2. During the reverse loading step the fraction of twinned microstructure decreases again. It has been shown that the stress which is required to nucleate a new twin is higher than the stress required to move a twin, i.e. propagate the twin boundaries to enlarge or shrink the twin $[6,17]$. Thus, untwinning of the microstructure during reverse loading, i.e. the shrinkage of existing twins and re-orientation to the original orientation of the parent grain is preferred over the nucleation and growth of new twins. This is consistent with the flow behaviour, because untwinning will result in the same work hardening behaviour as nucleation and growth of twins $[5,18,19]$. However, Fig. 5c shows that in some cases, where grains completely re-oriented as a result of twinning during the compression test segment, new twins nucleate with an orientation similar to that of the original untwinned grain.

The high AE count rates measured during the compression test segment in Fig. 1 and especially the fact that a significant fraction of the AE count rates are of burst type (indicated by the high count rate at the upper threshold level) are in full agreement with the ongoing nucleation of twins, especially at and after the yield point. It has been shown that twin growth itself is not a source of $\mathrm{AE}$ whereas the formation of new twins is [11]. This is very similar to the findings in an earlier paper [9] in which it was shown that twin growth without formation of new twins does not produce any detectable AE. The similarity between the mechanisms of twin growth and twin shrinkage (or untwinning) allow the assumption to be made, that the untwinning mechanism will also not produce AE [11]. Thus, untwinning is seen as the dominant mechanism in the test after compression to $125 \mathrm{MPa}$, because the formation of new twins would reveal $\mathrm{AE}$ in the tension segment of the test. In Fig. $1 \mathrm{~b}$ and $\mathrm{c}$, at higher compressive stress, considerable $\mathrm{AE}$ count rates are found in the subsequent tensile test segments at both threshold levels. Very similar results were presented in work by Lou et al. [5], where also a considerable amount of $\mathrm{AE}$ is found during a tensile test segment after pre-compression. Thus, a change in the underlying mechanism in these experiments can be assumed and this mechanism has to be a source of AE. In this case the formation of new twins in twinned grains is a very likely explanation for this effect. Although the resulting count rates are rather low, this corresponds well with the fact that only some grains show these newly nucleated twins and in fact, this mechanism does not control the overall microstructural evolution. It is noteworthy that these twins occur in grains of intermediate size, i.e. not in the very large grains that are typically not completely twinned and also not in very small grains.

In an earlier paper it was shown that twins are preferentially activated in larger grains in the case of a distinct grain size distribution [9]. After reaching a maximum number of twins in the microstructure, the twinned fraction of the microstructure increases, i.e. the twins grow. This finding is confirmed in the present work where larger grains preferentially twin during the compressive test segment and only at higher compressive stresses do smaller grains also twin with a more distinct frequency. It is noted that often in the large grains a larger number of twins form at the same time which is typically not found in smaller grains. Thus, grains of intermediate size can completely re-orient during the compressive test segment whereas larger grains still show fractions of the untwinned parent grain.

Re-orientation of grains during the tensile test segment is first visible in smaller grains that more frequently do not show twins any more whereas smaller twins are still visible in larger grains. It can be assumed that twins in larger grains had more time to grow because of earlier nucleation during the compression test and therefore require higher tensile stress levels to untwin completely again, whereas this condition can be reached earlier in smaller grains.

\section{Summary}

EBSD measurement and AE measurements confirm the impact of $\{10-12\}\langle 10-1-1\rangle$ twins during compression of an extruded AZ31 magnesium alloy. The preferential activation is understood by the strong texture of the sample which favours twinning in compression along the extrusion axis. Twins preferentially form in larger grains. It requires higher compressive stress levels to form twins also in smaller grains. During a reverse loading tensile test segment, untwinning is a significant mechanism which leads to the same features as twinning in the work hardening behaviour of the material as the original nucleation and growth of twins do. No AE count rates are produced after application of low compressive stresses which corresponds to the fact that twin growth and shrinkage are not sources of AE. At higher stress levels, considerable $\mathrm{AE}$ is detected which corresponds to 
the formation of new twins in already twinned grains. AE count rates are low as the frequency of this mechanism is also low. Smaller grains untwin preferentially whereas twins are still found in larger grains after tensile testing.

\section{Acknowledgments}

The authors would like to thank Dr. Peter Beaven at the Helmholtz-Zentrum Geesthacht for discussions on the topic of this work. This work received support from the Czech Science Foundation under grant P108/ $10 /$ P430. K.P. is grateful for financial support from the Grant Agency of Charles University No. 166310 and also for the grant SVV-2011-263303, and F.C. is grateful to the Alexander von Humboldt Stiftung for financial support during his stay in Germany.

\section{References}

[1] M.R. Barnett, Z. Keshavarz, A.G. Beer, D. Atwell, Acta Mater. 52, 5093 (2004).

[2] C.H.J. Davies, S. Yi, J. Bohlen, K.U. Kainer, H.G. Brokmeier, Mater. Sci. Forum 495-497, 1633 (2005).

[3] J. Bohlen, S.B. Yi, J. Swiostek, D. Letzig, H.G. Brokmeier, K.U. Kainer, Scr. Mater. 53, 259 (2005).

[4] Y.N. Wang, J.C. Huang, Acta Mater. 55, 897 (2007).

[5] X.Y. Lou, M. Li, R.K. Boger, S.R. Agnew, R.H. Wagoner, Int. J. Plasticity 23, 44 (2007).

[6] P.G. Partridge, Metall. Rev. 118, 169 (1967).
[7] J. Bohlen, P. Dobron̆, J. Swiostek, D. Letzig, F. Chmelík, P. Lukáč, K.U. Kainer, Mater. Sci. Eng. A 462, 302 (2007).

[8] M.A. Meyers, O. Vohringer, V.A. Lubarda, Acta Mater. 49, 4025 (2001).

[9] P. Dobroň, F. Chmelík, S. Yi, K. Parfenenko, D. Letzig, J. Bohlen, Scr. Mater. 65, 424 (2011).

[10] L. Nascimento, L. Fuskova, J. Bohlen, D. Letzig, K.U. Kainer, in: Proc. 8th Int. Conf. on Magnesium Alloys and Their Applications, Weimar, Elsevier, Weinheim 2010, p. 639.

[11] C.R. Heiple, S.H. Carpenter, J. Acoustic Emission 6, 177 (1987).

[12] J. Bohlen, P. Dobron̆, J. Swiostek, D. Letzig, F. Chmelík, P. Lukáč, K.U. Kainer, Mater. Sci. Eng. A 462, 307 (2007).

[13] Standard Practice for Acoustic Emission Examination of Fibre Glass Reinforced Plastic Resin, ASTM E 1067-85, Tank/Vessels, May 31, 1985.

[14] J. Bohlen, F. Chmelík, P. Dobroň, D. Letzig, F. Kaiser, P. Lukáč, K.U. Kainer, J. Alloys Comp. 378, 214 (2004).

[15] I.L. Dillamore, W.T. Roberts, Metall. Rev. 10, 271 (1965).

[16] E.W. Kelly, W.F. Hosford, Trans. Metall. Soc. AIME 242, 5 (1968).

[17] R.E. Reed Hill, R. Abbaschian, Physical Metallurgy Principles, PWS Publ. Company, Boston 1994.

[18] S. Kleiner, P.J. Uggowitzer, Mater. Sci. Eng. A 379, 258 (2004).

[19] M.A. Gharghouri, G.C. Weatherly, J.D. Embury, J. Root, Philos. Mag. A 79, 1671 (1999). 